OPEN ACCESS

Edited by:

Fu Wang,

Xi'an Jiaotong University, China

Reviewed by:

Fengbiao Mao,

University of Michigan, United States

Chaoyun Pan

Sun Yat-Sen University, China

*Correspondence:

Ping $\mathrm{Hu}$

lyzxyyhp@163.com

Specialty section:

This article was submitted to

Precision Medicine,

a section of the journal

Frontiers in Medicine

Received: 04 December 2020 Accepted: 15 March 2021 Published: 13 April 2021

Citation:

Ge M, Niu J, Hu P, Tong A, Dai Y, Xu F and Li F (2021) A Ferroptosis-Related Signature Robustly Predicts Clinical

Outcomes and Associates With Immune Microenvironment for Thyroid Cancer. Front. Med. 8:637743.

doi: 10.3389/fmed.2021.637743

\section{A Ferroptosis-Related Signature Robustly Predicts Clinical Outcomes and Associates With Immune Microenvironment for Thyroid Cancer}

\author{
Mingqin Ge, Jie Niu, Ping Hu*, Aihua Tong, Yan Dai, Fangjiang Xu and Fuyuan Li \\ Department of Endocrinology, Linyi Central Hospital, Linyi, China
}

Objective: This study aimed to construct a prognostic ferroptosis-related signature for thyroid cancer and probe into the association with tumor immune microenvironment.

Methods: Based on the expression profiles of ferroptosis-related genes, a LASSO cox regression model was established for thyroid cancer. Kaplan-Meier survival analysis was presented between high and low risk groups. The predictive performance was assessed by ROC. The predictive independency was validated via multivariate cox regression analysis and stratified analysis. A nomogram was established and verified by calibration curves. The enriched signaling pathways were predicted via GSEA. The association between the signature and immune cell infiltration was analyzed by CIBERSORT. The ferroptosis-related genes were validated in thyroid cancer tissues by immunohistochemistry and RT-qPCR.

Results: A ferroptosis-related eight gene model was established for predicting the prognosis of thyroid cancer. Patients with high risk score indicated a poorer prognosis than those with low risk score $(p=1.186 \mathrm{e}-03)$. The AUCs for 1-, 2-, and 3-year survival were $0.887,0.890$, and 0.840 , respectively. Following adjusting other prognostic factors, the model could independently predict the prognosis $(p=0.015, \mathrm{HR}: 1.870,95 \% \mathrm{Cl}$ : 1.132-3.090). A nomogram combining the signature and age was constructed. The nomogram-predicted probability of 1-, 3-, and 5-year survival approached the actual survival time. Several ferroptosis-related pathways were enriched in the high-risk group. The signature was distinctly associated with the immune cell infiltration. After validation, the eight genes were abnormally expressed between thyroid cancer and control tissues.

Conclusion: Our findings established a prognostic ferroptosis-related signature that was associated with the immune microenvironment for thyroid cancer.

Keywords: ferroptosis, gene signature, thyroid cancer, immune microenvironment, prognosis

\section{INTRODUCTION}

Thyroid cancer is the most often diagnosed endocrine malignancy, accounting for $1 \%$ of all newly diagnosed cancers (1). In the past 30 years, the global incidence of thyroid cancer has markedly increased (2). The disease is expected to become the fourth major cancer worldwide (3). Surgery followed by radioactive iodine or observation is the main therapy for most of patients (2). 
The application of high-throughput technology is increasing, which deepens the understanding about the molecular characteristics of thyroid cancer. Molecular markers have also become effective tools for predicting prognosis and identifying new therapeutic targets in thyroid cancer management.

Activated immune cells in the tumor microenvironment secrete pro-inflammatory cytokines and chemokines, which may promote the progression of thyroid cancer (4). Immunotherapy provides new possibilities for curing thyroid cancer. Recently, the crosstalk between ferroptosis and immune cells is involved in mediating response to the immunotherapy (5). Ferroptosis characterized by unique morphologies (decreased cell size, elevated mitochondrial membrane density) and bioenergy characteristics, is a novel form of regulated cell death with an iron-dependent manner (6). Glutathione depletion or glutathione peroxidase 4 inactivation may lead to the metabolic imbalance, thereby inducing ferroptosis of cancer cells (7). Ferroptosis has exhibited great potential in cancer therapy. FDA has approved small molecule inducers of ferroptosis to kill cancer cells such as Erastin, Sulphasalazine, Sorafenib and Statins (8). However, the exact mechanisms of ferroptosis and the association with the tumor immune microenvironment have not been uncovered. Recently, Liang et al. established a robust prognostic gene signature based on 10 ferroptosis-related genes for hepatocellular carcinoma (9). Furthermore, Wu et al. developed a ferroptosis-related five signature for predicting prognosis for clear cell renal cell carcinoma (10). Nevertheless, at present, there is still a lack of ferroptosis-related gene model for predicting the prognosis of patients with thyroid cancer. Hence, in this study, we established a prognostic ferroptosis-related signature, which could robustly predict the survival time of patients and was associated with the immune microenvironment for thyroid cancer.

\section{MATERIALS AND METHODS}

\section{Thyroid Cancer Datasets}

RNA sequencing (RNA-seq) transcriptome data of thyroid cancer were downloaded from The Cancer Genome Atlas (TCGA, http://cancergenome.nih.gov/) database on September 14, 2020. Using the Ensembl database (http://asia.ensembl. org/index.html), gene names were transformed from Ensembl ID into gene symbol matrix. The corresponding clinical information including age, gender, stage and TNM was also retrieved from TCGA database. Following removing samples without complete follow-up information, 510 thyroid cancer samples and 58 normal samples were included in this study. Table 1 listed the clinical characteristics of these thyroid cancer patients. Sixty ferroptosis-related genes were obtained from the previous literature (9). The expression profiles of these

\footnotetext{
Abbreviations: RNA-seq, RNA sequencing; TCGA, The Cancer Genome Atlas; LASSO, least absolute shrinkage and selection operator; ROC, Receiver Operating Characteristic; PCA, Principal Component Analysis; GSEA, Gene Set Enrichment Analysis; FDR, false discovery rate; qRT-PCR, quantitative real-time PCR; HR, hazard ratio; $\mathrm{CI}$, confidence interval; AUC, area under the curve.
}

ferroptosis-related genes were extracted from above samples (Supplementary Table 1). Differentially expressed ferroptosisrelated genes were screened between thyroid cancer and normal samples utilizing the edgeR package in $\mathrm{R}$ with the threshold of adjusted $p<0.05$ (11).

\section{Construction of a Least Absolute Shrinkage and Selection Operator Cox Model}

Differentially expressed ferroptosis-related genes were used for construction of a LASSO Cox model. Prognosis-related genes were evaluated by univariate Cox regression analysis via the survival package in $\mathrm{R}$. Genes with $p<0.05$ were selected for LASSO Cox model analysis. A LASSO Cox model was then established through the Glmnet package in R (12). Variable selection was presented to get better performance parameters, followed by regularization so as to avoid overfitting. The regularization of LASSO was controlled by the parameter $\lambda$. The larger the $\lambda$, the greater the penalty for linear models with more variables. In this study, a 10 -fold cross-validation was presented for each $\lambda$ and the partial likelihood deviance values was determined. The optimal $\lambda$ was selected to establish the model. The risk score for each sample was calculated according to $\beta_{1} \mathrm{x}_{1}+\beta_{2} \mathrm{x}_{2}+\ldots+\beta_{\mathrm{p}} \mathrm{x}_{\mathrm{p}}$ (where $\beta_{\mathrm{p}}$ indicates the coefficients, and $x_{\mathrm{p}}$ indicates the gene expression levels). Based on the median value of risk scores, patients were separated into high- and low- risk groups. Kaplan-Meier overall survival (OS) analysis was presented, followed by log-rank test. The sensitivity and accuracy of the signature was validated by the Receiver Operating Characteristic (ROC) curve utilizing the SurvivalROC package in R. Principal Component Analysis (PCA) was presented for assessing the accuracy of the classification according to the high and low risk scores.

\section{Univariate and Multivariate Cox Regression Analysis}

Univariate cox regression analysis was presented for assessment of the prognostic values of the risk score and clinical features (age, gender, stage, T, N, and M). Afterwards, multivariate cox regression analysis was used to determine which prognostic factors could independently predict the survival of patients.

\section{Nomogram Model Establishment}

By combining the eight ferroptosis-related genes, a nomogram was established. Moreover, another nomogram was constructed by combining age and the risk score. The total point of each patient was calculated based on the nomogram. The nomogrampredicted probability of 1-, 3-, and 5-year survival time was contrast by the actual survival time, which was visualized by the calibration curves.

\section{Stratified Survival Analysis}

Patients were divided into different subgroups according to age $(<65$ and $\geq 65$ ), gender (female and male) and stage (stage I-II 
TABLE 1 | The clinical characteristics of thyroid cancer patients.

\begin{tabular}{|c|c|c|}
\hline Characteristics & & Number \\
\hline \multicolumn{3}{|l|}{ Age } \\
\hline & $<65$ & 436 \\
\hline & $>65$ & 71 \\
\hline \multicolumn{3}{|l|}{ Gender } \\
\hline & Male & 136 \\
\hline & Female & 371 \\
\hline \multicolumn{3}{|l|}{ Stage } \\
\hline & Stage I & 285 \\
\hline & Stage \| & 52 \\
\hline & Stage III & 113 \\
\hline & Stage IV & 55 \\
\hline & Unknown & 2 \\
\hline \multicolumn{3}{|l|}{$\mathbf{T}$} \\
\hline & T1 & 144 \\
\hline & T2 & 167 \\
\hline & Т3 & 171 \\
\hline & T4 & 23 \\
\hline & Tx & 2 \\
\hline \multicolumn{3}{|l|}{ M } \\
\hline & MO & 283 \\
\hline & M1 & 9 \\
\hline & $M x$ & 213 \\
\hline & Unknown & 1 \\
\hline
\end{tabular}

and stage III-IV). Kaplan-Meier survival analysis followed by logrank test was presented between high and low risk score groups in different subgroups.

\section{Gene Set Enrichment Analysis}

GSEA was performed between high and low risk score groups (13). The "c2.cp.kegg.v7.1.symbols" was utilized as the reference. Signaling pathways with nominal $p$-value $<0.05$ and false discovery rate $($ FDR $)<0.05$ were significantly enriched.

\section{Estimation of Immune Infiltration}

The infiltration levels of 22 immune cells in thyroid cancer samples were assessed via CIBERSORT (http://cibersort. stanford.edu/) (14). The differences in the infiltration levels of immune cells were compared between high and low risk score groups via the Wilcoxon rank-sum-test.

\section{Immunohistochemistry}

Immunohistochemistry of DPP4, GPX4, GSS, AKR1C1, HMGCR, TFRC, SQLE, and PGD in thyroid cancer and normal tissues was obtained from The Human Protein Atlas database (https://www.proteinatlas.org/).

\section{Patients and Specimens}

Twenty thyroid cancer and 10 adjacent normal tissues were obtained from Linyi Central Hospital between 2019 and 2020. Thyroid cancer was confirmed by post-operative pathological
TABLE 2 | Primer sequences for qRT-PCR

\begin{tabular}{|c|c|}
\hline Primer & Sequence $\left(5^{\prime}-3^{\prime}\right)$ \\
\hline \multirow[t]{2}{*}{ DPP4 } & АTTCCAAACAACACACAGT (forward) \\
\hline & CTTCATAAACCCAGTCAGT (reverse) \\
\hline \multirow[t]{2}{*}{ GPX4 } & GTAAAACCGGACCAGAAGTACAAG (forward) \\
\hline & CCCACCTGCTTCCCGAACTG (reverse) \\
\hline \multirow[t]{2}{*}{ GSS } & CTGGAGCGGCTGAAGGACA (forward) \\
\hline & AGCTCTGAGATGCACTGGACA (reverse) \\
\hline \multirow[t]{2}{*}{ AKR1C1 } & TGCAGAGGTTCCTAAAAGTAAAGCTITA (forward) \\
\hline & GGAAAATGAATAAGGTAGAGGTCAACATAA (reverse) \\
\hline \multirow[t]{2}{*}{ HMGCR } & AGTTTGAAGAGGATGTITG (forward) \\
\hline & TCCCTTACTTCATCCTGTGA (reverse) \\
\hline \multirow[t]{2}{*}{ TFRC } & ACCATTGTCATATACCCGGTTCA (forward) \\
\hline & CAATAGCCCAAGTAGCCAATCAT (reverse) \\
\hline \multirow[t]{2}{*}{ SQLE } & TGGTTACATGATTCATGATC (forward) \\
\hline & TACTGAACTCCCATCACAAC (reverse) \\
\hline \multirow[t]{2}{*}{ PGD } & АTTCTCAAGTTCCAAGACACCG (forward) \\
\hline & GTGGTAAAACAGGGCATGGGA (reverse) \\
\hline \multirow[t]{2}{*}{ GAPDH } & CTGCCCAGAACATCATCC (forward) \\
\hline & CTCAGATGCCTGCTTCAC (reverse) \\
\hline
\end{tabular}

examination. Before surgery, no patient underwent radiation therapy or thyrotropin suppression therapy. The study was approved by the Ethics Committee of Linyi Central Hospital and followed the Declaration of Helsinki (2019019). Each patient provided written informed consent. All the resected specimens were placed instantly into liquid nitrogen and stored at $-80^{\circ} \mathrm{C}$.

\section{Quantitative Real-Time PCR}

Total RNA was extracted from tissues or cells via TRIzol (Invitrogen, Carlsbad, California, USA), followed by reverse transcription into cDNA. PCR was carried out using the TB Green ${ }^{\circledR}$ Premix Ex Taq ${ }^{\mathrm{TM}}$ II kit (TAKARA, China). The reaction procedures were as follows: 40 cycles at $94^{\circ} \mathrm{C}$ lasting $15 \mathrm{~s}, 60^{\circ} \mathrm{C}$ lasting $10 \mathrm{~s}$, and $72^{\circ} \mathrm{C}$ lasting $20 \mathrm{~s}$. GAPDH served as an internal control. The relative expression levels were quantified with the $2^{-\Delta \Delta C t}$ method. The primer sequences were listed in Table 2.

\section{Cell Culture and Transfection}

Human thyroid cancer cell lines TPC-1 and FTC-133 (American Type Culture Collection, ATCC) were cultured in Dulbecco's modified eagle medium (Gibco, USA) containing 10\% FBS, $100 \mathrm{U} / \mathrm{ml}$ penicillin and $100 \mu \mathrm{g} / \mathrm{ml}$ streptomycin in a $5 \% \mathrm{CO}_{2}$ incubator at $37^{\circ} \mathrm{C}$. The cells were seeded in 6 -well plates $(2 \times$ $10^{5} /$ well). When the cell confluence reached $60 \%$, transfection of siRNA-negative control (si-NC), si-AKR1C1\#1 and siAKR1C1\#2 was carried out according to the Lipofectamine 2000 (Invitrogen, USA) instructions. After $48 \mathrm{~h}$, the cells were harvested.

\section{Flow Cytometry}

The transfected cells were seeded in 6 -well plates $(2 \times$ $10^{4} /$ well). After culturing for $48 \mathrm{~h}$, the culture medium was discarded, and the cells were trypsinized and collected. 


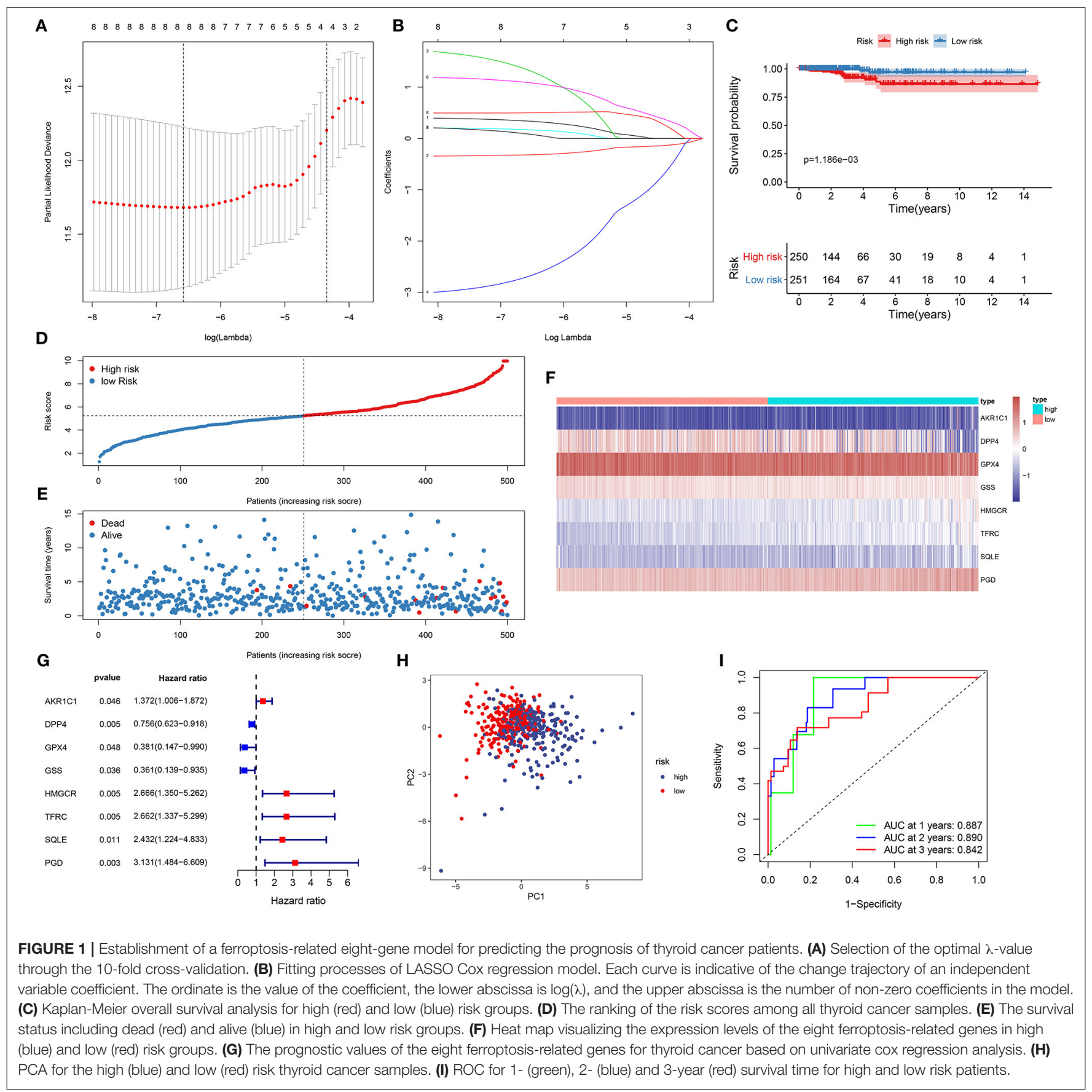

Following the instructions of Annexin V-FITC / PI kit, 200 $\mu \mathrm{l}$ binding buffer was added to resuspend cells, followed by $5 \mu \mathrm{l}$ Annexin V-FITC and $5 \mu \mathrm{l}$ PI. After incubating for $20 \mathrm{~min}$ in the dark, the apoptosis rate was detected by flow cytometry (BD, USA).

\section{Wound Healing}

The transfected cells were seeded into 24 -well plates $(5 \times$ $10^{4}$ /well). The next day, a $200 \mu \mathrm{l}$ pipette tip was utilized to draw a straight line perpendicular to the bottom of the cell culture plate.
The width of scratches $(\times 40)$ was investigated at 0 and $24 \mathrm{~h}$ under a microscope.

\section{Transwell}

Matrigel was diluted at 1:10 by serum-free medium, which was added to the upper layer of the transwell chamber (Corning, USA). The transfected cells diluted to $2 \times 10^{5} / \mathrm{ml}$ with serumfree medium were added to the upper layer of the chamber at 100 $\mu l$ per well. Five hundred microliter of medium containing $10 \%$ FBS was added to the lower layer. After $24 \mathrm{~h}$, the cells were fixed 


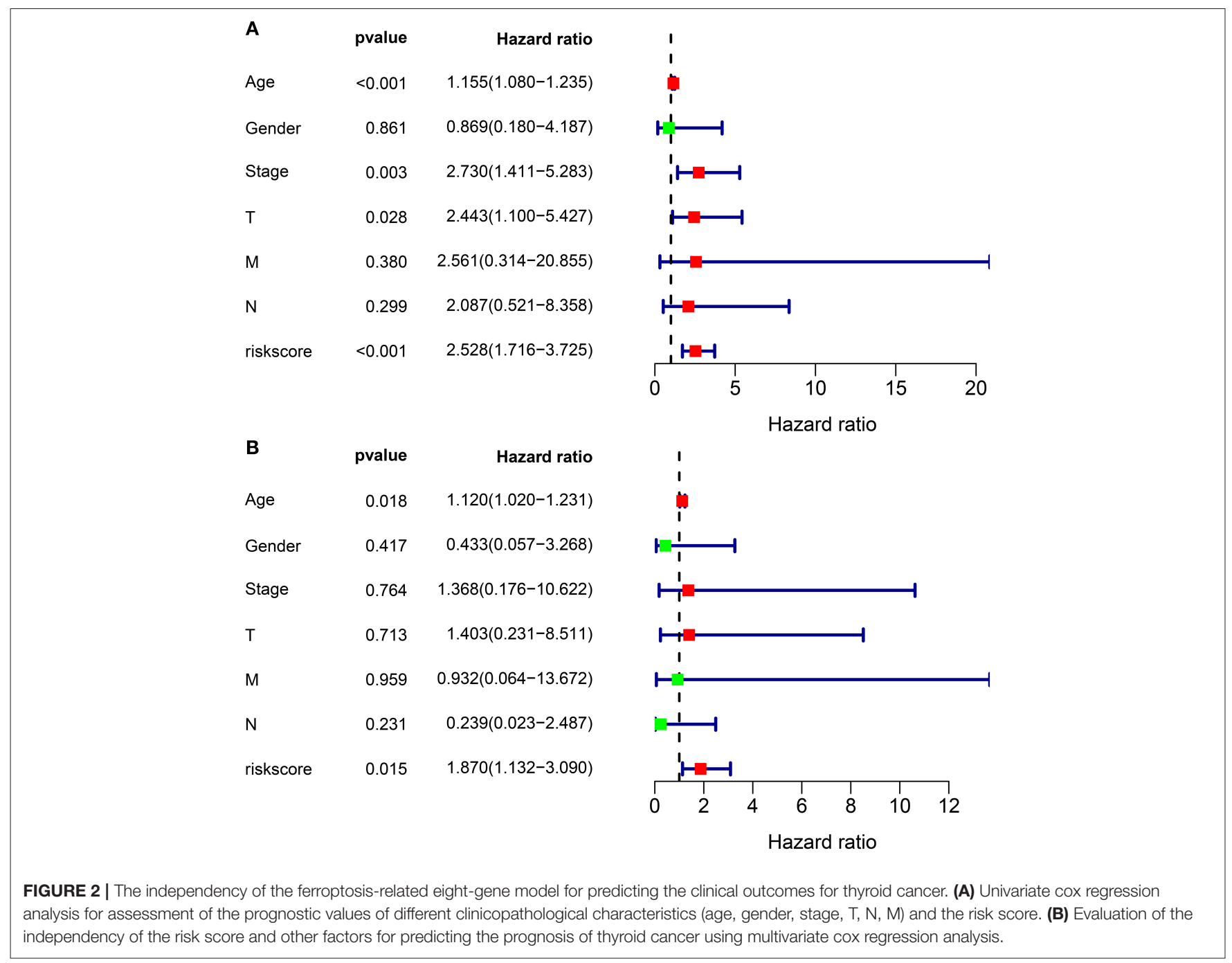

with formaldehyde and stained with crystal violet. The number of invasive cells was counted.

\section{Statistical Analysis}

Statistical analysis was presented through R 3.6.3 and GraphPad 7.0. Data were presented as means \pm standard deviation. Comparisons between two groups were analyzed by paired student's $t$-test or Wilcoxon rank-sum-test. Multiple comparisons were presented by one-way ANOVA. Difference with $p<0.05$ was considered statistically significant.

\section{RESULTS}

\section{Differentially Expressed Ferroptosis-Related Genes for Thyroid Cancer}

A total of 60 ferroptosis-related genes were included in this study. Among them, 46 genes with adjusted $p<0.05$ were differentially expressed between 510 thyroid cancer samples and 58 normal samples.

\section{Establishment of a Prognostic Ferroptosis-Related Eight-Gene Model for Thyroid Cancer}

Based on 46 differentially expressed ferroptosis-related genes, prognosis-related genes were selected for LASSO Cox regression analysis. When $\log \lambda=-6.6$, the model exhibited the optimal performance and the least number of independent variables (Figure 1A). As the values of $\lambda$ increased, the LASSO coefficients of these variables were close to zero (Figure 1B). As a result, eight ferroptosis-related genes were utilized for the establishment of a prognostic model. The risk score for each sample was calculated as follows: (0.340143834733433) * AKR1C1 expression + $(-0.319305763663027) *$ DPP4 expression $+(1.3413362890122)$ * GPX4 + (-2.69880844893117) * GSS + (0.171053637326744)* HMGCR + (1.0923479137719)* TFRC + (0.0892997114590679) * SQLE + $(0.499540011822522) *$ PGD. In line with the 


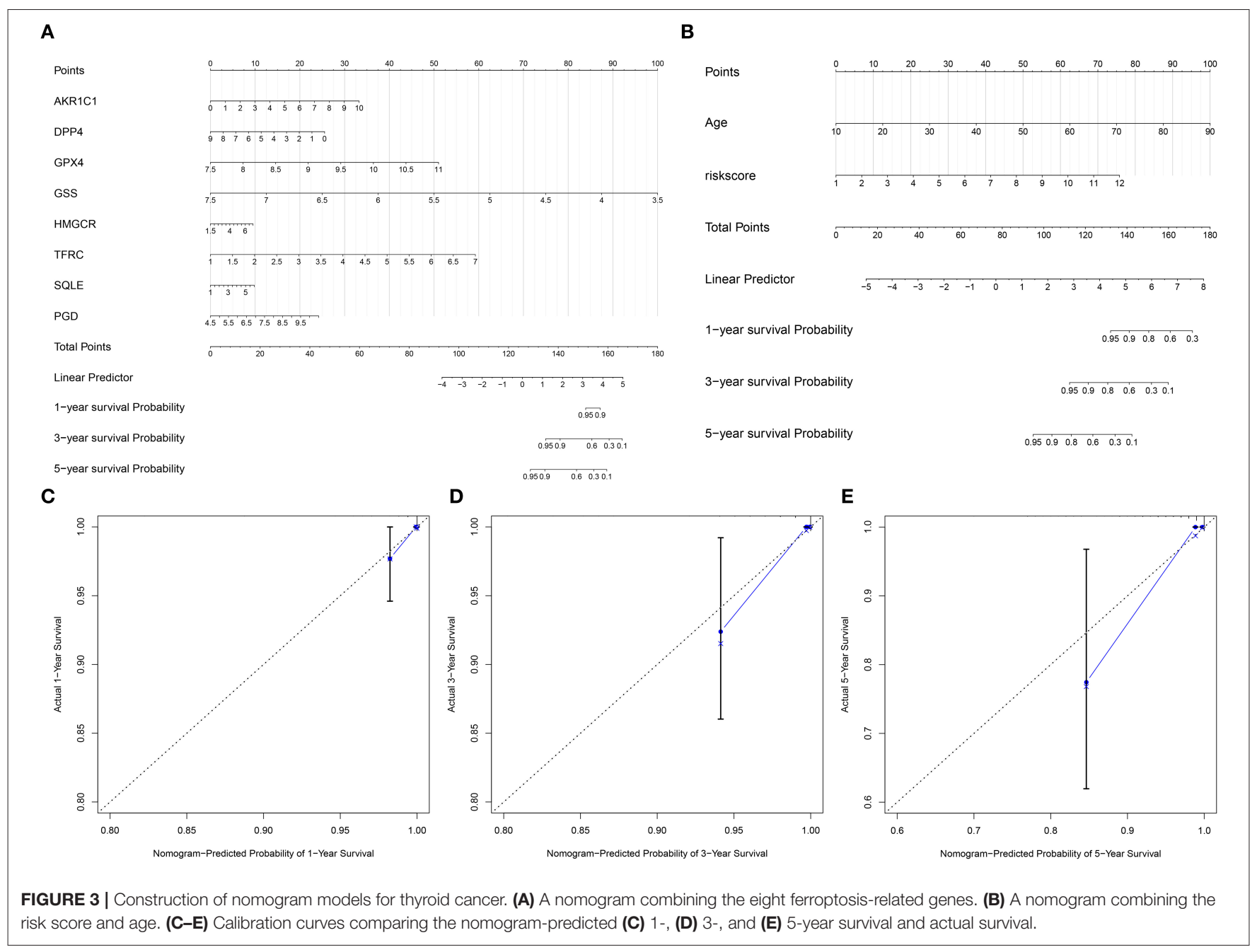

median value of all risk scores, 510 thyroid cancer patients were separated into high- and low-risk score groups. KaplanMeier curves showed that low risk patients could outlive high risk patients 21 ( $p=1.186-03$; Figure 1C). As the risk scores increased, the number of died patients was gradually increased (Figures 1D,E). Heat map visualized the expression patterns of the eight ferroptosis-related genes between highand low-risk groups (Figure 1F). Univariate cox regression analysis demonstrated that DPP4 [hazard ratio (HR): 0.756, 95\% confidence interval (CI): 0.623-0.918, $p=0.004$ ], GPX4 (HR: 0.381, 95\% CI: $0.147-0.990, p=0.048$ ) and GSS (HR: $0.361,95 \%$ CI: $0.139-0.935, p=0.036)$ were protective factors for thyroid cancer prognosis (Figure 1G). Meanwhile, AKR1C1 (HR: 1.372, 95\% CI: $1.006-1.872, p=0.046$ ), HMGCR (HR: 2.666, 95\% CI: $1.350-5.262, p=0.005)$, TFRC (HR: 2.662, 95\% CI: 1.337-5.299, $p=0.005)$, SQLE (HR: 2.432, 95\% CI: 1.224-4.833, $p=0.011)$ and PGD (HR: 3.131, 95\% CI: 1.484-6.609, $p=0.003$ ) were risk factors for thyroid cancer prognosis (Figure 1G). PCA results confirmed the accuracy of the classification among the thyroid cancer samples (Figure 1H). To further validate the accuracy and sensitivity of the model, we constructed a ROC analysis. Our data confirmed that the model could accurately and sensitively predict the survival probability for 1- [area under the curve $(\mathrm{AUC})=0.887], 2-(\mathrm{AUC}=0.890)$, and 3-year $(\mathrm{AUC}=0.842)$ survival time (Figure 1I). Collectively, this ferroptosis-related eight-gene model could be a robust prognostic model for thyroid cancer.

\section{The Ferroptosis-Related Eight-Gene Model Was an Independent Factor for Predicting Prognosis of Thyroid Cancer}

We further evaluated the performance of the ferroptosis-related eight-gene model for predicting the prognosis of thyroid cancer patients. Firstly, univariate cox regression analysis results showed that the risk score was a risk factor for thyroid cancer prognosis (HR: 2.528, 95\% CI: 1.716-3.725, $p<0.001$ ) in Figure 2A. Furthermore, age (HR: 1.155, 95\% CI: 1.080-1.235, $p<0.001$ ), stage (HR: 2.730, 95\% CI: 1.411-5.283, $p=0.003$ ), and $\mathrm{T}$ (HR: 2.443 , 95\% CI: $1.100-5.427, p=0.028$ ) were significantly associated with thyroid cancer prognosis (Figure 2A). Following multivariate cox regression analysis, the risk score was an 


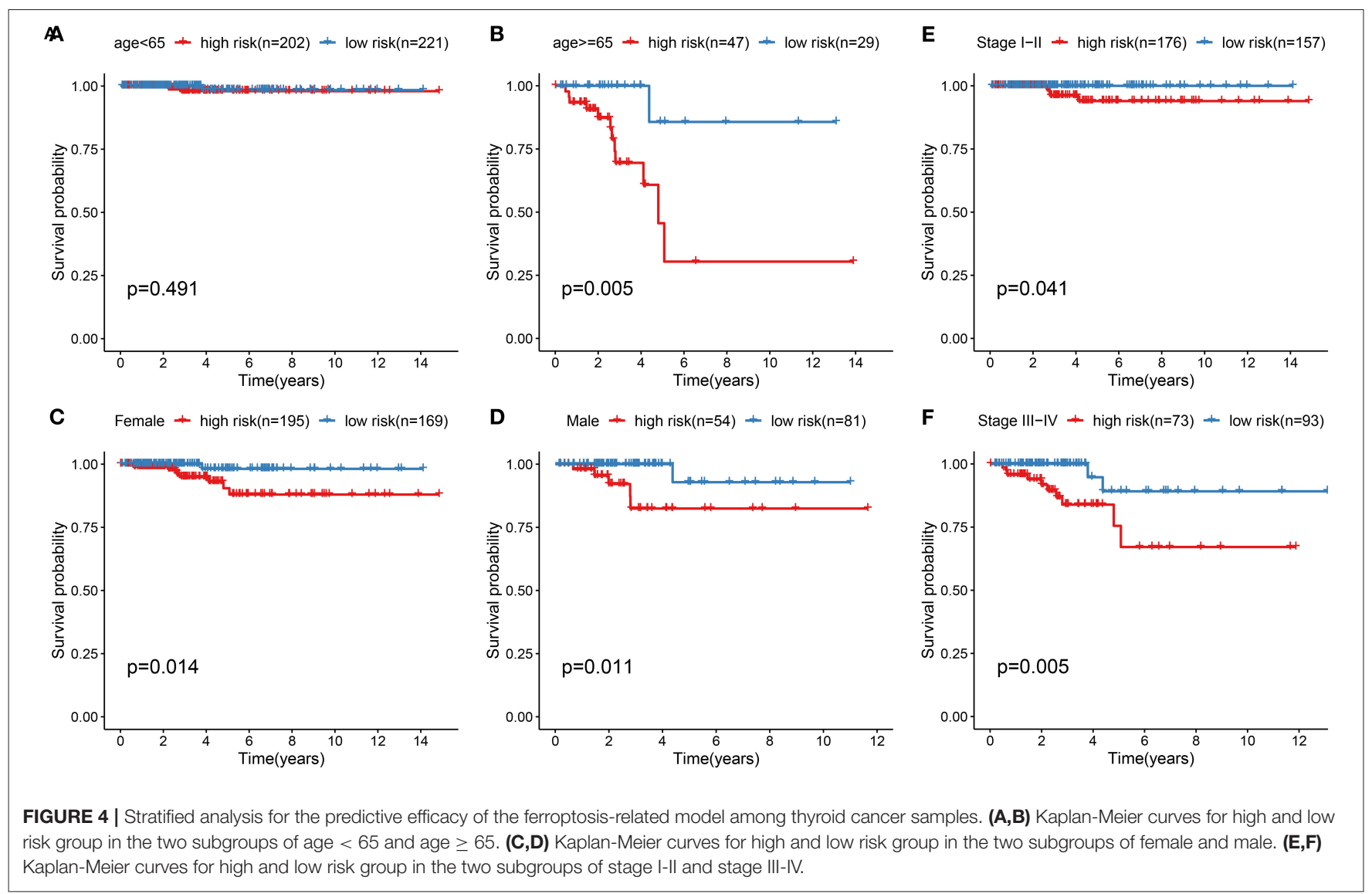

independent risk factor for thyroid cancer (HR: 1.870, 95\% CI: 1.132-3.090, $p=0.015$; Figure 2B). Except to the risk score, age independently predicted the prognosis of thyroid cancer (HR: 1.120, 95\% CI: 1.020-1.231, $p=0.018$ ). Collectively, the ferroptosis-related eight-gene model was an independent prognostic factor for thyroid cancer.

\section{Construction of Nomogram Models for Thyroid Cancer}

On the basis of the eight ferroptosis-related genes that could independently predict the prognosis of thyroid cancer, a nomogram was established for predicting 1-, 3-, and 5-year survival probability of thyroid cancer patients (Figure 3A). Furthermore, by combining the two independent prognostic factors (age and risk score), we constructed a nomogram model for thyroid cancer (Figure 3B). Calibration curves confirmed that the nomogram-predicted 1- (Figure 3C), 3- (Figure 3D), and 5- (Figure 3E) year survival probability approached the actual survival.

\section{Stratified Analysis for the Predictive Efficacy of the Ferroptosis-Related Model}

To assess whether the ferroptosis-related eight-gene model could sensitively predict the prognosis of thyroid cancer, stratified analysis was performed. For patients with age $<65$, there was no significant difference in the overall survival time between high and low risk groups (Figure 4A). For patients aged $>65$, high risk score was indicative of the shorter survival time compared to low risk score (Figure 4B; $p=0.005$ ). Both for female (Figure 4C; $p=0.014$ ) and male (Figure 4D; $p=0.011$ ) patients, high risk score suggested a poorer prognosis in comparison to low risk score. In Figure 4E, patients at the stage I-II in the high-risk group exhibited the less optimistic prognosis than those in the low-risk group $(p=0.041)$. Similarly, high risk score indicated the poorer clinical outcomes for patients with stage III-IV than low risk score (Figure 4F; $p=0.005$ ). Collectively, the model was a sensitive prognostic marker for thyroid cancer.

\section{Potential Signaling Pathways for High-Risk Group}

To uncover the potential signaling pathway in high- and lowrisk groups, we presented GSEA. Our GSEA results showed that calcium signaling pathway $(\mathrm{NES}=1.8147893$ and $p<$ 0.0001 ), MAPK signaling pathway (NES $=1.7950739$ and $p=$ $0.0042643924)$, mTOR signaling pathway $(\mathrm{NES}=1.8197428$ and $p<0.0001)$, pathways in cancer $(\mathrm{NES}=1.7282541$ and $p=$ $0.008281574)$, PPAR signaling pathway $(\mathrm{NES}=1.7024437$ and $p$ $=0.008247423)$, TGF-beta signaling pathway $(\mathrm{NES}=1.8606039$ and $p=0.0020283975$ ), and WNT signaling pathway (NES = 1.8473499 and $p=0.0020876827$ ) were significantly enriched in the high-risk group (Figure 5). However, no pathways with 

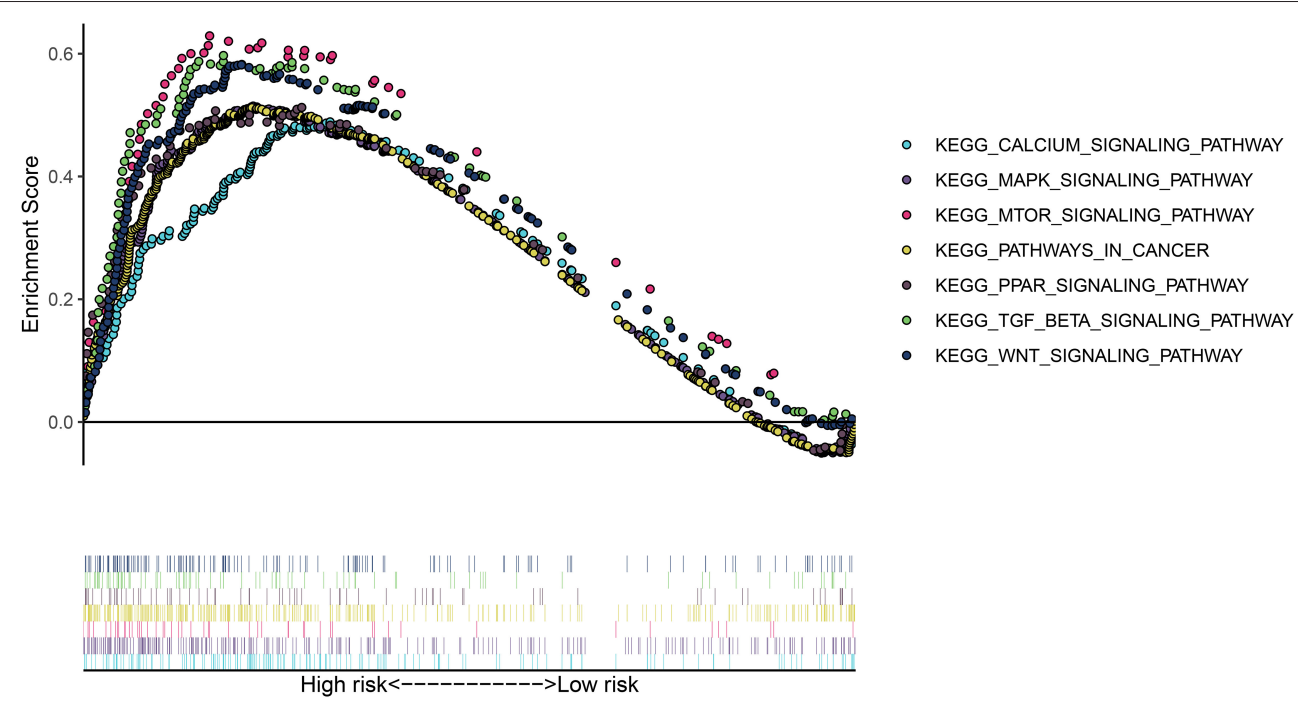

FIGURE 5 | GSEA revealed the signaling pathways enriched in the high-risk group.

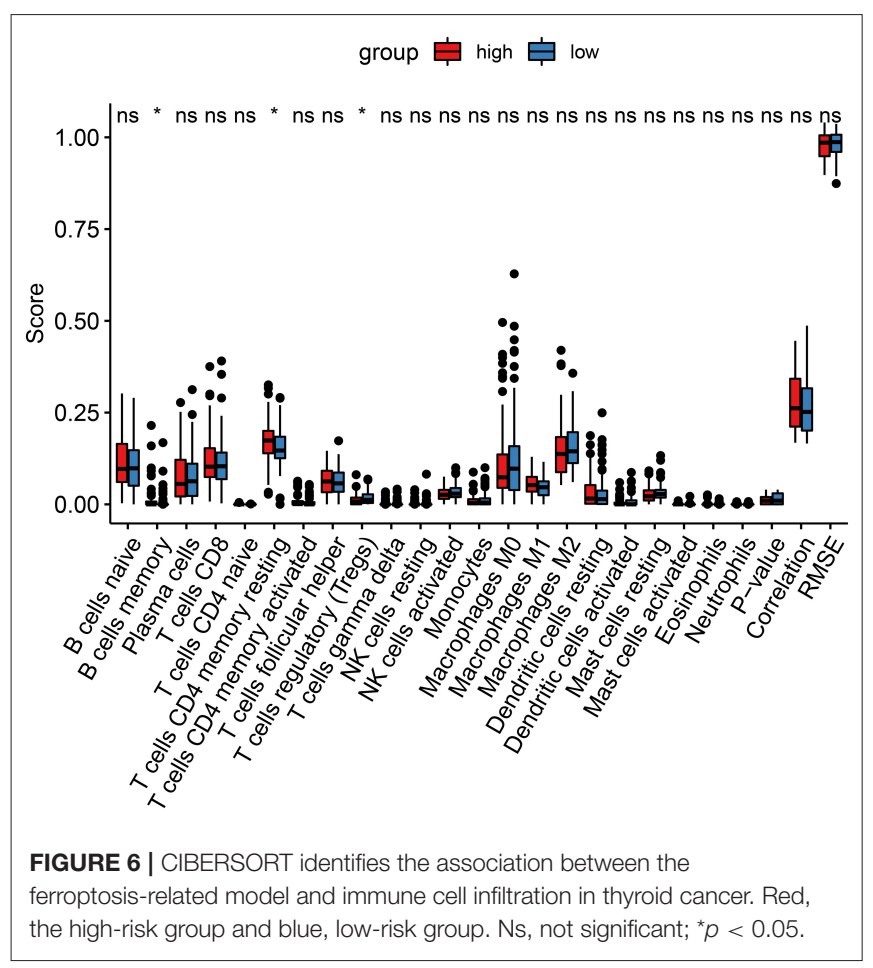

nominal $p$-value $<0.05$ and FDR $<0.05$ were found in the lowrisk group, indicating that there were no pathways significantly enriched in the low-risk group.

\section{The Ferroptosis-Related Model Is Associated With Immune Cell Infiltration in Thyroid Cancer}

It has been reported that ferroptosis is involved in the tumor immune microenvironment. Hein, we assessed the correlation between the ferroptosis-related risk scores and immune cell infiltrations among thyroid cancer patients using the CIBERSORT. In Figure 6, the risk score was significantly associated with the infiltration levels of B cells memory $(p<0.05)$, T cells CD4 memory resting $(p<0.05)$, and T cells regulatory (Treg; $p<0.05$ ). The high-risk samples exhibited a distinctly higher infiltration level of T cells CD4 memory resting compared to the low risk samples. Lower infiltration level of Tregs was detected in the high-risk group than the low risk group.

\section{Validation of Eight Ferroptosis-Related Genes in Thyroid Cancer Tissues}

We further validated the expression of the eight ferroptosisrelated genes in thyroid cancer and control tissues. Immunohistochemistry results showed the expression and distribution of AKR1C1 (Figure 7A), DPP4 (Figure 7B), GPX4 (Figure 7C), GSS (Figure 7D), HMGCR (Figure 7E), TFRC (Figure 7F), SQLE (Figure 7G), and PGD (Figure 7H) in thyroid cancer and normal tissues. Furthermore, the mRNA expression levels of the eight ferroptosis-related genes were examined between thyroid cancer and normal tissues by RTqPCR. Our data suggested that AKR1C1 ( $p<0.0001$; Figure 8A), DPP4 ( $p<0.0001$; Figure 8B), GPX4 ( $p=0.0001$; Figure 8C), GSS $(p<0.0001$; Figure 8D), HMGCR $(p<0.0001$; Figure 8E), TFRC ( $p<0.0001$; Figure 8F), SQLE ( $p=0.0004$; Figure 8G), and PGD ( $p=0.0005$; Figure $\mathbf{8 H}$ ) were all significantly highly expressed in thyroid cancer tissues compared to normal tissues.

\section{Silencing AKR1C1 Promotes Apoptosis and Suppresses Migration and Invasion in Thyroid Cancer Cells}

Among the eight ferroptosis-related genes, we selected AKR1C1 to validate its function in thyroid cancer. AKR1C1 expression was distinctly silenced by si-AKR1C1 in TPC-1 and FTC-133 cells (Figure 9A). After silencing its expression, the apoptotic 


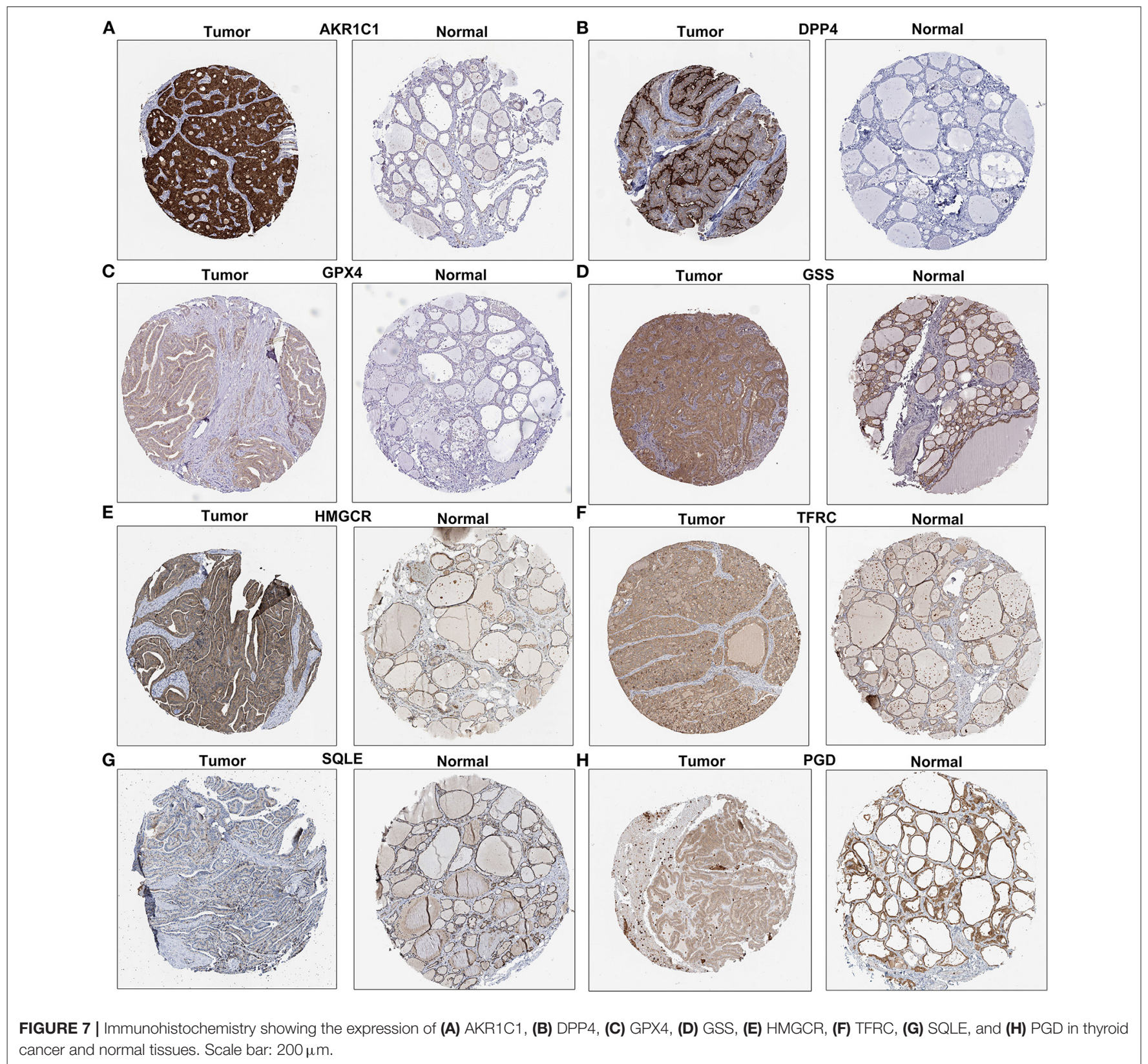

levels were significantly promoted in TPC- 1 and FTC-133 cells (Figures 9B,C). Also, we found that the migrated (Figures 9D,E) and invasive (Figures 9F,G) capacities were markedly restrained by AKR1C1 knockdown in TPC-1 and FTC-133 cells. These data demonstrated that AKR1C1 might participate in thyroid cancer progression.

\section{DISCUSSION}

Increasing evidence emphasizes the importance of ferroptosis on the cancer therapy. Nevertheless, it remains unclear whether ferroptosis may affect the clinical outcomes for thyroid cancer patients. Thus, elucidating the molecular mechanisms and signaling pathways of ferroptosis enables us to develop novel therapeutic targets for thyroid cancer. In this study, we developed a ferroptosis-related eight gene model, which can robustly predict the prognosis of thyroid cancer.

Herein, a ferroptosis-related eight gene model was constructed for predicting the prognosis of thyroid cancer via LASSO Cox regression analysis. For thyroid cancer patients, high risk score was indicative of poorer prognosis than low risk score. ROC confirmed that the signature could have accurate and sensitive predictive efficacy. Multivariate cox regression analysis demonstrated that the signature can independently predict the survival for thyroid cancer. Among them, DPP4, GPX4, and GSS were protective factors for thyroid cancer prognosis. Meanwhile, AKR1C1, HMGCR, TFRC, SQLE, and PGD were risk factors for thyroid cancer prognosis. As previous studies, DPP4 is highly expressed in thyroid cancer tissues, which has been considered 

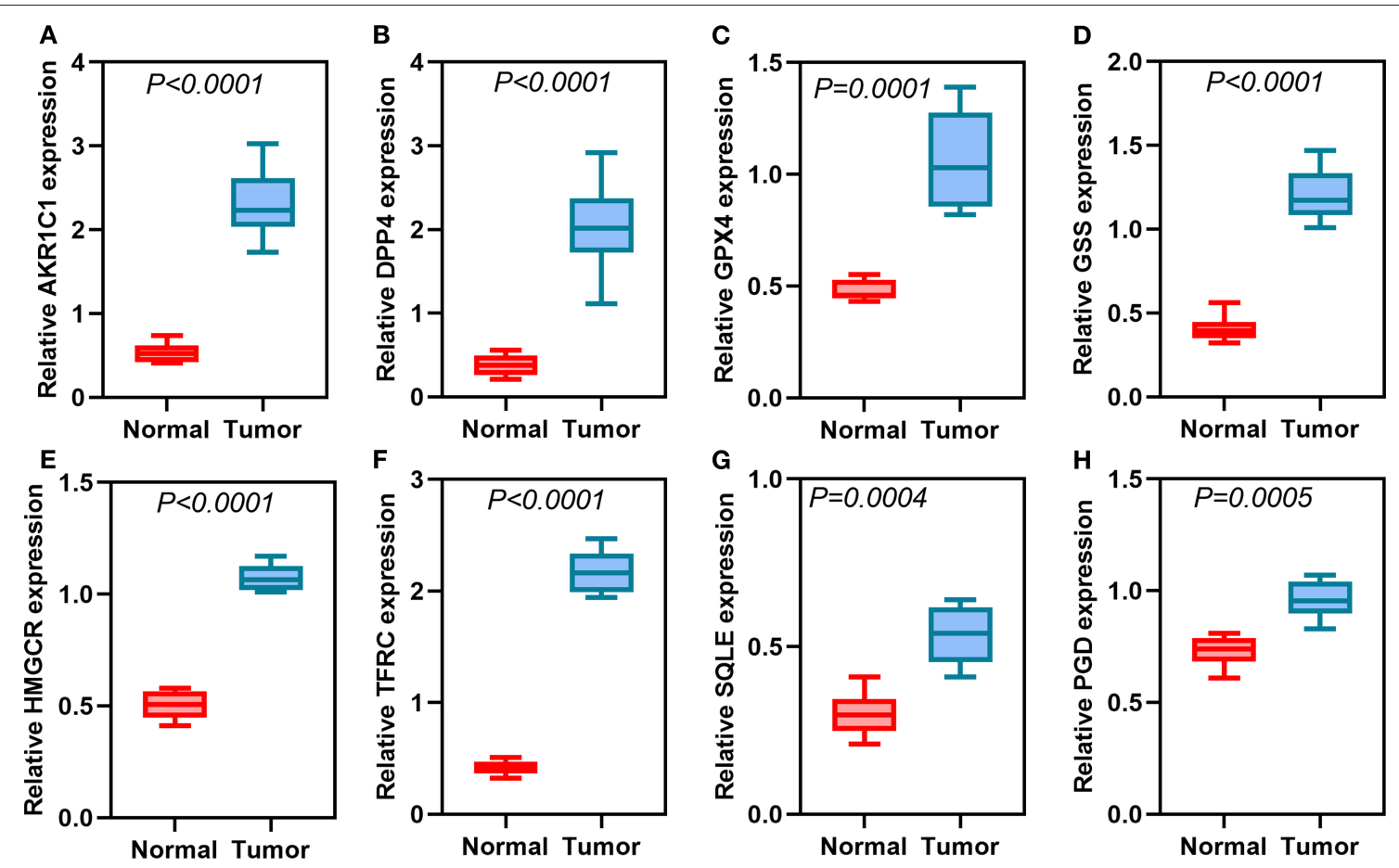

FIGURE 8 | RT-qPCR detecting the mRNA expression of (A) AKR1C1, (B) DPP4, (C) GPX4, (D) GSS, (E) HMGCR, (F) TFRC, (G) SQLE, and (H) PGD in thyroid cancer and normal tissues.

as a prognostic factor (15). It can induce proliferative, invasive, as well as migrated capacities for thyroid cancer (16). GPX4 is an important regulator of ferroptosis for cancer cells (17). Depletion of GPX4 may induce ferroptosis, which can sensitize cells to ferroptosis (18). AKR1C1 can activate STAT3, thereby promoting lung cancer metastasis (19). Furthermore, it mediates cisplatin-resistance for head and neck squamous cell carcinoma via STAT1/3 pathways (20). Targeting HMGCR may suppress the invasive ability of thyroid cancer cells (21). It has been reported that YAP can facilitate ferroptosis via up-regulation of a ferroptosis regulator TFRC in cancer cells (22). Moreover, TFRC induces epithelial ovarian cancer cell proliferation as well as metastases by up-regulating AXIN2 (23). SQLE has become a pharmaceutical target for various cancers. For example, it may drive non-alcoholic fatty liver disease-induced hepatocellular carcinoma (24). Additionally, SQLE expression can predict the fatality rate of prostate cancer (25). However, the functions of these eight ferroptosis-related genes remains to be clarified in thyroid cancer. Our immunohistochemistry and qRT-PCR confirmed that these eight genes were all activated in thyroid cancer. This study may offer novel clues for exploring the biological roles and clinical significance of these ferroptosisrelated genes. Our multivariate cox regression analysis suggested that age at diagnosis was an independent risk factor for thyroid cancer. Consistently, age has been commonly applied as a risk factor for stratifying the prognosis of thyroid cancer (26). Among adults aged over 65 years old, the incidence of thyroid cancer is the highest (27). Our stratified analysis revealed that the ferroptosis-related eight gene model could accurately predict the clinical outcomes for patients with $>65$ years old. Moreover, regardless of whether it was a male or female patient, high risk score implied a worse prognosis. For patients with stage I-II or III-IV, high risk score was indicative of shorter survival time. Hence, the signature could accurately predict the clinical outcomes for thyroid cancer. In order to expand the clinical application of this model, we combined it with age to construct a nomogram that could be easy to calculate the expected survival rate for an individual patient. Calibration curves confirmed that the nomogram-predicted 1-, 3-, and 5-year survival time was close to the actual survival time. This suggested that the nomogram had the potential as a clinically predictive tool for thyroid cancer prognosis.

Various signaling pathways participate in regulating the process of ferroptosis. Herein, our data suggested that MAPK, mTOR, pathways in cancer, PPAR, TGF-beta, and WNT signaling pathways were enriched in the high-risk group. MAPK pathway can be activated in ferroptosis cells (28). In turn, blockage of MAPK pathway also protects cells from ferroptosis (29). The activation of MAPK pathways can lead to the occurrence and progression of thyroid cancer (30). Ferroptosis-induced ROS accumulation could inactivate MAPK pathway to kill thyroid cancer cells (30). Four targeted therapies (Sorafenib, Lenvatinib, Vandetanib, and Cabozantinib) have been approved for treating thyroid cancer patients resistant to standard therapy, which can have the effects via blockage of MAPK pathway (31). Hence, their capacity to prolong the survival time of patients remain to be limited because of poor efficacies and other molecular interventions such as ferroptosis (31). mTOR pathway 


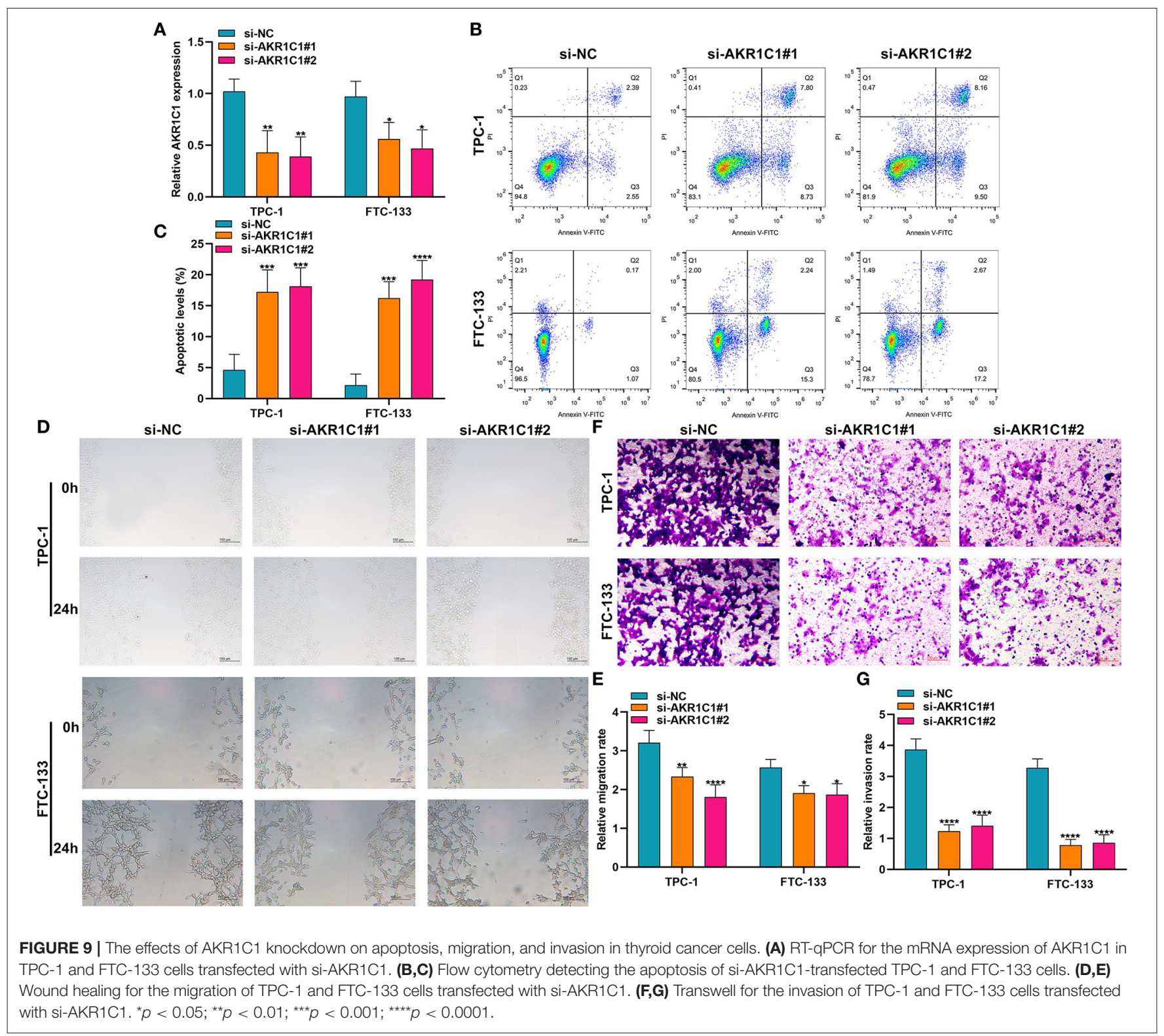

can mediate cellular proliferative capacities and the uptake of iodine for thyroid cells (32). Recently, mTOR pathway has been confirmed to be involved in mediating ferroptosis in different cancers such as colorectal cancer (33). Activation of mTOR transduction may protect cancer cells from oxidative stress and ferroptosis (34). PPAR $\alpha$ promotes ferroptosis by mediating lipid remodeling in cancer cells (35). In WNT pathway, Frizzled-7 is sensitive to ferroptosis for platinum-tolerant ovarian cancer cells (36). Taken together, our results indicated that various pathways could be involved in ferroptosis for thyroid cancer. The roles of these pathways in ferroptosis should be assessed by more experiments.

Many thyroid cancer patients who are not accompanied by thyroiditis, immune infiltrations are found following surgery, suggesting that changes in the immune microenvironment is related to the progression of thyroid cancer (37). Our data demonstrated that the model was significantly associated with the infiltration of B cells memory, T cells CD4 memory resting and Tregs. Further analysis should be presented to probe into the associations between the ferroptosis-related model and the tumor microenvironment. A larger cohort should be required to verify the predictive values of the ferroptosis-related eight gene model for thyroid cancer.

\section{CONCLUSION}

Collectively, we constructed a ferroptosis-related eight gene model that exhibited a well predictive performance. The model had a significant association with the tumor immune microenvironment. The nomogram combining the model and age could provide new possibilities for individualized therapy of thyroid cancer patients. Hence, ferroptosis could be promising therapeutic targets. 


\section{DATA AVAILABILITY STATEMENT}

The datasets presented in this study can be found in online repositories. The names of the repository/repositories and accession number(s) can be found in the article/Supplementary Material.

\section{ETHICS STATEMENT}

The studies involving human participants were reviewed and approved by the Ethics Committee of Linyi Central Hospital (2019019). The patients/participants provided their written informed consent to participate in this study.

\section{REFERENCES}

1. Hernando J, Ros J, Arroyo A, Capdevila J. Clinical and translational challenges in thyroid cancer. Curr Med Chem. (2020) 27:480622. doi: 10.2174/0929867327666200214125712

2. Cabanillas ME, McFadden DG, Durante C. Thyroid cancer. Lancet. (2016) 388:2783-95. doi: 10.1016/S0140-6736(16)30172-6

3. Kim J, Gosnell JE, Roman SA. Geographic influences in the global rise of thyroid cancer. Nat Rev Endocrinol. (2020) 16:17-29. doi: 10.1038/s41574-019-0263-x

4. Varricchi G, Loffredo S, Marone G, Modestino L, Fallahi P, Ferrari SM, et al. The immune landscape of thyroid cancer in the context of immune checkpoint inhibition. Int J Mol Sci. (2019) 20:3934. doi: 10.3390/ijms20163934

5. Wang W, Green M, Choi JE, Gijón M, Kennedy PD, Johnson JK, et al. CD8(+) $\mathrm{T}$ cells regulate tumour ferroptosis during cancer immunotherapy. Nature. (2019) 569:270-4. doi: 10.1038/s41586-019-1170-y

6. Shen Z, Song J, Yung BC, Zhou Z, Wu A, Chen X. Emerging strategies of cancer therapy based on ferroptosis. Adv Mater. (2018) 30:e1704007. doi: 10.1002/adma.201704007

7. Lu B, Chen XB, Ying MD, He QJ, Cao J, Yang B. The role of ferroptosis in cancer development and treatment response. Front Pharmacol. (2017) 8:992. doi: 10.3389/fphar.2017. 00992

8. Hassannia B, Vandenabeele P, Vanden Berghe T. Targeting ferroptosis to iron out cancer. Cancer Cell. (2019) 35:830-49. doi: 10.1016/j.ccell.2019. 04.002

9. Liang JY, Wang DS, Lin HC, Chen XX, Yang H, Zheng Y, et al. A novel ferroptosis-related gene signature for overall survival prediction in patients with hepatocellular carcinoma. Int J Biol Sci. (2020) 16:243041. doi: $10.7150 /$ ijbs. 45050

10. Wu G, Wang Q, Xu Y, Li Q, Cheng L. A new survival model based on ferroptosis-related genes for prognostic prediction in clear cell renal cell carcinoma. Aging (Albany NY). (2020) 12:14933-48. doi: 10.18632/aging.103553

11. Robinson MD, McCarthy DJ, Smyth GK. edgeR: a bioconductor package for differential expression analysis of digital gene expression data. Bioinformatics. (2010) 26:139-40. doi: 10.1093/bioinformatics/btp616

12. Friedman J, Hastie T, Tibshirani R. Regularization paths for generalized linear models via coordinate descent. J Stat Softw. (2010) 33:1-22. doi: 10.18637/jss. v033.101

13. Subramanian A, Tamayo P, Mootha VK, Mukherjee S, Ebert BL, Gillette MA, et al. Gene set enrichment analysis: a knowledge-based approach for interpreting genome-wide expression profiles. Proc Natl Acad Sci USA. (2005) 102:15545-50. doi: 10.1073/pnas.0506580102

14. Newman AM, Liu CL, Green MR, Gentles AJ, Feng W, Xu Y, et al. Robust enumeration of cell subsets from tissue expression profiles. Nat Methods. (2015) 12:453-7. doi: 10.1038/nmeth.3337

\section{AUTHOR CONTRIBUTIONS}

PH conceived and designed the study. MG, JN, and AT conducted most of the experiments and data analysis, and wrote the manuscript. YD, FX, and FL participated in collecting data and helped to draft the manuscript. All authors reviewed and approved the manuscript.

\section{SUPPLEMENTARY MATERIAL}

The Supplementary Material for this article can be found online at: https://www.frontiersin.org/articles/10.3389/fmed. 2021.637743/full\#supplementary-material

Supplementary Table 1 | A list of 60 ferroptosis-related genes.

15. Du J, Fu L, Ji F, Wang C, Liu S, Qiu X. FosB recruits KAT5 to potentiate the growth and metastasis of papillary thyroid cancer in a DPP4-dependent manner. Life Sci. (2020) 259:118374. doi: 10.1016/j.lfs.2020.118374

16. Wang Y, Han J, Lv Y, Zhang G. miR-29a inhibits proliferation, invasion, and migration of papillary thyroid cancer by targeting DPP4. Onco Targets Ther. (2019) 12:4225-33. doi: 10.2147/OTT.S201532

17. Yang WS, SriRamaratnam R, Welsch ME, Shimada K, Skouta R, Viswanathan VS, et al. Regulation of ferroptotic cancer cell death by GPX4. Cell. (2014) 156:317-31. doi: 10.1016/j.cell.2013.12.010

18. Chen GQ, Benthani FA, Wu J, Liang D, Bian ZX, Jiang X. Artemisinin compounds sensitize cancer cells to ferroptosis by regulating iron homeostasis. Cell Death Differ. (2020) 27:24254. doi: 10.1038/s41418-019-0352-3

19. Zhu H, Chang LL, Yan FJ, Hu Y, Zeng CM, Zhou TY, et al. AKR1C1 activates STAT3 to promote the metastasis of non-small cell lung cancer. Theranostics. (2018) 8:676-92. doi: 10.7150/thno.21463

20. Chang WM, Chang YC, Yang YC, Lin SK, Chang PM, Hsiao M. AKR1C1 controls cisplatin-resistance in head and neck squamous cell carcinoma through cross-talk with the STAT1/3 signaling pathway. J Exp Clin Cancer Res. (2019) 38:245. doi: 10.1186/s13046-019-1256-2

21. Revilla G, Pons MP, Baila-Rueda L, García-León A, Santos D, Cenarro A, et al. Cholesterol and 27-hydroxycholesterol promote thyroid carcinoma aggressiveness. Sci Rep. (2019) 9:10260. doi: 10.1038/s41598-019-46727-2

22. Wu J, Minikes AM, Gao M, Bian H, Li Y, Stockwell BR, et al. Intercellular interaction dictates cancer cell ferroptosis via NF2-YAP signalling. Nature. (2019) 572:402-6. doi: 10.1038/s41586-019-1426-6

23. Huang Y, Huang J, Huang Y, Gan L, Long L, Pu A, et al. TFRC promotes epithelial ovarian cancer cell proliferation and metastasis via up-regulation of AXIN2 expression. Am J Cancer Res. (2020) 10:131-47.

24. Liu D, Wong CC, Fu L, Chen H, Zhao L, Li C, et al. Squalene epoxidase drives NAFLD-induced hepatocellular carcinoma and is a pharmaceutical target. Sci Transl Med. (2018) 10:aap9840. doi: 10.1126/scitranslmed.aap9840

25. Stopsack KH, Gerke TA, Sinnott JA, Penney KL, Tyekucheva S, Sesso HD, et al. Cholesterol metabolism and prostate cancer lethality. Cancer Res. (2016) 76:4785-90. doi: 10.1158/0008-5472.CAN-16-0903

26. Shen X, Zhu G, Liu R, Viola D, Elisei R, Puxeddu E, et al. Patient age-associated mortality risk is differentiated by BRAF V600E status in papillary thyroid cancer. J Clin Oncol. (2018) 36:438-45. doi: 10.1200/JCO.2017.74.5497

27. Haymart MR, Banerjee M, Reyes-Gastelum D, Caoili E, Norton EC. Thyroid ultrasound and the increase in diagnosis of low-risk thyroid cancer. J Clin Endocrinol Metab. (2019) 104:785-92. doi: 10.1210/jc.2018-01933

28. Nakamura T, Naguro I, Ichijo H. Iron homeostasis and iron-regulated ROS in cell death, senescence and human diseases. Biochim Biophys Acta Gen Subj. (2019) 1863:1398-409. doi: 10.1016/j.bbagen.2019.06.010

29. Poursaitidis I, Wang X, Crighton T, Labuschagne C, Mason D, Cramer $\mathrm{SL}$, et al. Oncogene-selective sensitivity to synchronous cell death following modulation of the amino acid nutrient cystine. Cell Rep. (2017) 18:254756. doi: 10.1016/j.celrep.2017.02.054 
30. Su X, Shen Z, Yang Q, Sui F, Pu J, Ma J, et al. Vitamin C kills thyroid cancer cells through ROS-dependent inhibition of MAPK/ERK and PI3K/AKT pathways via distinct mechanisms. Theranostics. (2019) 9:446173. doi: 10.7150/thno.35219

31. Naoum GE, Morkos M, Kim B, Arafat W. Novel targeted therapies and immunotherapy for advanced thyroid cancers. Mol Cancer. (2018) 17:51. doi: 10.1186/s12943-018-0786-0

32. Souza EC, Ferreira AC, Carvalho DP. The mTOR protein as a target in thyroid cancer. Expert Opin Ther Targets. (2011) 15:1099-112. doi: 10.1517/14728222.2011.594044

33. Zhang L, Liu W, Liu F, Wang Q, Song M, Yu Q, et al. IMCA induces ferroptosis mediated by SLC7A11 through the AMPK/mTOR pathway in colorectal cancer. Oxid Med Cell Longev. (2020) 2020:1675613. doi: 10.1155/2020/1675613

34. Yi J, Zhu J, Wu J, Thompson CB, Jiang X. Oncogenic activation of PI3K-AKTmTOR signaling suppresses ferroptosis via SREBP-mediated lipogenesis. Proc Natl Acad Sci USA. (2020) 117:31189-97. doi: 10.1073/pnas.2017152117

35. Venkatesh D, O’Brien NA, Zandkarimi F, Tong DR, Stokes ME, Dunn DE, et al. MDM2 and MDMX promote ferroptosis by PPAR $\alpha$-mediated lipid remodeling. Genes Dev. (2020) 34:526-43. doi: 10.1101/gad.334219.119
36. Wang Y, Zhao G, Condello S, Huang H, Cardenas H, Tanner EJ, et al. Frizzled-7 identifies platinum tolerant ovarian cancer cells susceptible to ferroptosis. Cancer Res. (2020) 384-99. doi: 10.1101/2020.05.28. 121590

37. Nicolson NG, Brown TC, Korah $\mathrm{R}$, Carling $\mathrm{T}$. Immune cell infiltrate-associated dysregulation of DNA repair machinery may predispose to papillary thyroid carcinogenesis. Surgery. (2020) 167:66-72. doi: 10.1016/j.surg.2019.02.024

Conflict of Interest: The authors declare that the research was conducted in the absence of any commercial or financial relationships that could be construed as a potential conflict of interest.

Copyright (c) $2021 \mathrm{Ge}$, Niu, Hu, Tong, Dai, Xu and Li. This is an open-access article distributed under the terms of the Creative Commons Attribution License (CC BY). The use, distribution or reproduction in other forums is permitted, provided the original author(s) and the copyright owner(s) are credited and that the original publication in this journal is cited, in accordance with accepted academic practice. No use, distribution or reproduction is permitted which does not comply with these terms. 